\title{
La determinación económica, social y laboral de la enfermedad renal crónica de causas no tradicionales (ERCnT) en la costa sur de Guatemala
}

\author{
The economic, social and labor determination of chronic kidney disease of non-traditional \\ causes (CKDnT) on the southern coast of Guatemala \\ Sandra Herrera-Ruiz* \\ Facultad Latinoamericana de Ciencias Sociales, Guatemala
}

*Autor al que se dirige la correspondencia: sandelizaherrera@gmail.com

Recibido: 6 de marzo 2020 / Revisión: 14 de marzo 2020 / Aceptado: 29 de marzo 2020

\section{Resumen}

T a flexibilidad laboral es característica de la producción estacional agroindustrial, cuyo principal problema es Laprovechar con intensidad los períodos en los que se incrementa la producción. Pero, ¿cómo la demandante competitividad laboral influye en las estrategias de los trabajadores para incrementar su productividad? A partir de esta pregunta, se identificaron los aspectos económicos, sociales y laborales que inciden en la salud, así como las respuestas para disminuir el dolor físico y aumentar las exigencias de competitividad, flexibilización y desregularización del mercado de trabajo. La exposición ambiental y ocupacional en las condiciones de trabajo, el estrés térmico asociado a la deshidratación en la actividad física intensa, el limitado acceso a los servicios de salud, la mala alimentación y la pobre calidad de vida, también pueden producir otras enfermedades como diabetes e hipertensión, así como infecciones y lesiones renales. En ese contexto, en los últimos 20 años, se empezaron a reportar casos de enfermedad renal. La metodología cualitativa aplicada a este estudio, permitió llevar a cabo un proceso investigativo descriptivo e interpretativo sobre la forma en que los sujetos interactúan. Para ello se utilizaron las trayectorias laborales como técnicas en la recolección de datos. Los resultados arrojaron información relevante sobre las estrategias que los trabajadores utilizan para incrementar su rendimiento laboral, entre ellas la automedicación de vitaminas y analgésicos para tratar los espasmos musculares, que estimulan el sistema nervioso central, así como de bebidas saborizadas y energizantes con posibles repercusiones en la excreción renal.

Palabras claves: Condiciones de trabajo, flexibilización laboral, desregularización laboral, exposición ambiental, estrés térmico, excreción renal

\begin{abstract}
$\mathrm{L}$ abor flexibility is characteristic of seasonal agroindustrial production whose main problem is to take advantage

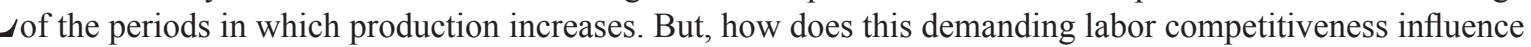
workers' strategies to increase their productivity? From this question, the economic, social and labor aspects that affect health were identified, as well as the answers to reduce physical pain and increase the demands for competitiveness, flexibility and deregulation of the labor market. Environmental and occupational exposure in working conditions, thermal stress associated with dehydration in intense physical activity, limited access to health services, poor diet and quality of life, can also cause other diseases such as diabetes and hypertension, as well as infections and kidney lesions. In that context, in the last 20 years, cases of kidney disease began to be reported. The qualitative methodology applied to this study, allowed to carry out a descriptive and interpretive investigation process on the way in which the subjects interact, for this, the work trajectories were used as techniques in data collection. The results yielded relevant information on the strategies that workers use to increase their work performance, including self-medication of "vitamins" and analgesics to treat muscle spasms, drugs that stimulate the central nervous system, as well as flavored and energizing drinks with possible repercussions on renal excretion.
\end{abstract}

Keywords Working conditions, labor flexibility, labor deregulation, environmental exposure, heat stress, renal excretion 


\section{Introducción}

En el año 2000, culminó el proceso de descampesinización en la costa sur y con ello la completa modificación del mercado laboral, impactando los volúmenes, usos de los empleos y las actividades laborales. La descampesinización ha sido abordada por la sociología del trabajo y por la antropología económica para explicar la forma en que las fuerzas económicas del mercado hacen que el campesino rompa su relación con la tierra y con el control de los procesos productivos que garantizan su sobrevivencia. En ese proceso se "incorporan territorios a dinámicas homogeneizadoras de productos exportables, con integración a circuitos comerciales cada vez más extensos, con mano de obra cada vez más prescindible" (Hocsman, 2015, p. 12).

Ahora son los consorcios agroindustriales (Prakash Sethi, 2003), los clústeres y encadenamientos productivos, los que generan empleos rurales directos e indirectos y quienes participan como fuerza laboral son profesionales, administrativos, técnicos y trabajadores agrícolas seleccionados por su productividad y competitividad.

Para la industria, la agricultura constituye un reto que solo es conquistado con programas intensivos de innovación tecnológica, científica y de desarrollo del capital social. Así la investigación en biotecnología, biogenética y climatología, forman parte de la lógica de excelencia empresarial. Sin embargo, desde sus raíces la producción agroindustrial ha necesitado grandes contingentes de trabajadores agrícolas que se emplean estacionariamente y que reaccionan al mercado laboral. Al respecto Samuelson y Nordhaus (2002) explican que hay un encuentro entre oferta y demanda, que no necesariamente llegan a coincidir.

La flexibilidad laboral es característica de la producción estacional agroindustrial (Fausto, 2017), por lo que en el moderno y asalariado mercado de trabajo el problema clave para empleadores y trabajadores es aprovechar con intensidad los períodos en los que se incrementa la demanda laboral. La oferta laboral queda, entonces, relegada a un segundo plano. Ante la desregulación laboral, el trabajador queda frente al empleador sin mediación del Estado, "libre de políticas sociales" (Roberts, 1998, p. 38) con contratos transitorios, adaptación de horarios laborales, y subcontrataciones que inciden en la falta de cobertura de la seguridad social en caso de enfermedad.

Con base a entrevistas a profundidad y trayectorias laborales, se analizan las condiciones de trabajo, la actividad física intensa y la exposición al calor sin sombra. Así como las estrategias de los trabajadores agrícolas para insertarse en los mercados laborales y corresponder a los incentivos por productividad, pero a cuenta del costo económico, social y de salud.

\section{Materiales y métodos}

La costa sur se caracteriza por concentrar la mayor cantidad de actividades agroindustriales de exportación, con aproximadamente 1,500 plantaciones ubicadas en más 180,000 ha cultivadas con caña de azúcar en los departamentos de Escuintla, Suchitepéquez, Retalhuleu y Santa Rosa, se conforma un circuito regional conectado en una lógica de productividad y competitividad guiado por la asociación empresarial que las aglutina.

Los más importantes ingenios azucareros están localizados en el departamento de Escuintla, específicamente en el municipio de Santa Lucía Cotzumalguapa y Siquinalá, mientras que una apreciable cantidad de plantaciones también están ubicadas en los municipios de Masagua, Tiquisate, La Gomera, La Democracia y la Nueva Concepción.

En el municipio de Santa Lucía Cotzumalguapa están ubicados cuatro ingenios azucareros y uno más en su área de influencia, es decir en Siquinalá. Es por ello que esta investigación focalizó su universo de estudio en los asentamientos urbanos de Santa Lucía Cotzumalguapa, que constituyen el reservorio habitacional de los trabajadores agroindustriales de la región.

Respecto a la unidad de análisis, los sujetos de la investigación fueron seleccionados con base al criterio que fueran trabajadores agrícolas. Por ello los entrevistados fueron únicamente hombres en edades comprendidas en tres rangos de edad: (1) mayores de 50 años (que durante toda su vida dependieron por completo de las actividades agrícolas de la caña de azúcar), (2) de 26 a 50 años (que se emplearon eventualmente en las actividades agrícolas o industriales de la caña de azúcar) y, (3) de 15 a 25 años (que tuvieron débil vinculación con las actividades agrícolas de la caña de azúcar).

Desde el método cualitativo (Taylor \& Bogdan, 1996), para llevar a cabo la recolección de información se diseñaron tres guías de entrevistas para trabajadores agrícolas que dieron como resultado: (1) Diez entrevistas exploratorias dirigidas a quienes fueron mozos colonos de rancherías, trabajadores en labores de campo, trabajadores permanentes, trabajadores técnicos y supervisores que realizaban trabajos especializados; (2) dos entrevistas a profundidad; y, (3) cinco trayectorias de vida familiar y laboral basadas en las nociones 
de temporalidad social, las cuales permitieron asir los elementos que dieron respuesta a los cambios laborales en la costa sur de Guatemala. Complementariamente se diseñó una guía de entrevista aplicada a tres representantes institucionales: del sector empresarial, supervisión de trabajo y supervisión de salud.

En total se llevaron a cabo 20 entrevistas hasta alcanzar la saturación de la información, haciendo un total de $69 \mathrm{~h}$ grabadas. Cada una de las cuatro guías de entrevista, fueron previamente validadas con otros trabajadores agrícolas que aceptaron colaborar con el estudio. El tipo y formato de los instrumentos de recolección de información permitió aplicar una estrategia metodológica replicable.

A esto se agrega el trabajo etnográfico, sobre la organización y forma de vida de los trabajadores agrícolas, con prolongadas estancias de campo que permitieron "ir hacia la gente y estar cara a cara con ellos" (Geertz, 1988). Complementariamente se utilizó información de un taller de discusión sobre aspectos de salud en Masagua, así como el análisis de discurso de los comunicados, memorias de labores y slogans institucionales, que en conjunto permitieron seguir la pista de los temas emergentes, desarrollo de conceptos y proposiciones para comenzar a dar sentido a los datos.

Todos los datos recolectados fueron codificados según las categorías diseñadas para este estudio, lo cual permitió registrar la frecuencia de información reiterativa, pero también de la sobrante, inesperada o aislada considerada como casos negativos a través de controles cruzados que permitieron verificar la coherencia o distorsión de los relatos. El análisis de la información se fundamentó en la intersubjetividad y comunicación lingüística (Foucault, 1968).

Finalmente se revisó la literatura relacionada con el tema, pera entender y explicar los resultados y correlacionar los diversos aspectos que son abordados en la Discusión.

\section{Resultados}

En los datos cualitativos proporcionados por 20 entrevistas realizadas en el área urbana del municipio de Santa Lucía Cotzumalguapa del departamento de Escuintla, fue posible indagar sobre la inserción e historial laboral, cualificación de la mano de obra, remuneración, patrones de residencia, agricultura de autoconsumo y atención de la salud. De esas entrevistas, 17 fueron aplicadas principalmente a trabajadores agroindustriales reportando que quienes tienen 50 años o más, crecieron en las fincas como mozos de rancherías semiasalariados, tienen escolaridad y experiencia laboral restringida, así como dificultades para reinsertarse en el mercado laboral agroindustrial completamente asalariado.

Por otro lado, quienes tienen entre 26 a 50 años reportan una mayor transición hacia el trabajo completamente asalariado, no obstante que oscilan entre el desempleo y subempleo, han logrado insertarse en el mercado laboral obteniendo con ello movilidad social. Por último, quienes tienen entre 15 a 25 años y una amplia gama de posibilidades incluyendo la migración a mercados laborales más atractivos. No obstante, cambian constantemente de empleo, por lo que existe una incompatibilidad entre trabajo, empleo y puesto laboral.

Es por ello que para incorporarse al mercado laboral y enfrentar la intensidad del trabajo reportaron una serie de estrategias, entre ellas el consumo de analgésicos muy potentes que llaman "vitaminas", pero también en casos extremos, el consumo de anfetaminas y psicotrópicos utilizados para trabajar sin dolor.

Los resultados arrojaron información sobre las estrategias para elevar su rendimiento laboral, y que están relacionadas con la automedicación de "vitaminas" (tiamina, inyecciones del complejo de las ocho vitaminas B y otros medicamentos que son analgésicos ante el dolor y que incrementan la energía celular). En faenas prolongadas, antes de iniciar los turnos de trabajo, se aprovisionan de fármacos que estimulan el sistema nervioso central como el modafinilo y los llamados popularmente como "ratones" (bebidas saborizadas energizantes con cafeína y taurina con efecto eufórico prolongado). Para disminuir el dolor por cansancio físico consumen pastillas que por su pequeño tamaño llaman "bebés" (tramadol) y fármacos derivados de la 1,4 benzodiazepina (para tratar los espasmos musculares).

Los efectos secundarios son el aumento de la frecuencia cardíaca y presión arterial. Aún no se conoce la incidencia directa sobre la salud de los trabajadores, pero se han identificado posibles repercusiones en los embarazos, diabetes mellitus y alcoholismo. Además, el uso prolongado crea adicción, afectando directamente el sistema nervioso y la excreción renal.

Durante la continuación del trabajo etnográfico en 2020, entre los vendedores ambulantes de los campos agrícolas llamados "chamorreros", se pudo constatar la venta libre de anti-inflamatorios no-esteroidaes (aines), bebidas energizantes, inhibidores del sueño que son excretados por vía renal y pueden estar relacionados con la etiología de la enfermedad.

Por otro lado, también se reporta que las entrevistas aplicadas a tres representantes del sector empre- 
sarial, supervisión de trabajo y supervisión de salud, complementar la información proporcionada por estos trabajadores agroindustriales.

Siempre relacionado con la situación de la costa sur, un taller de discusión en Masagua reportó casos aislados de leptospirosis que podrían sumarse al cuadro de deterioro de la salud de los trabajadores agrícolas de la región.

Por último es importante agregar que, si bien cada productor actúa con relativa independencia, existe una lógica operativa para todo el cordón azucarero respecto a lo que se entiende por calidad, productividad y competitividad, sobre los cambios tecnológicos, las características y eficiencia de los trabajadores, la responsabilidad empresarial con la salud y la educación, las alianzas público privadas y sobre las externalidades sociales en la región caracteriza por compartir elementos ambientales, económicos e históricos que la identifican como un poblamiento socio-cultural mestiza desde el siglo XVIII.

\section{Discusión}

\section{El trabajo en la determinación económica}

Para que los países agroexportadores se inserten en los inestables y volátiles mercados globales de los commodities, acuden a la desregulación y flexibilización laboral. Por ejemplo, en la costa sur "hasta mediados del siglo XX se animaba la voluminosa migración" de la fuerza de trabajo principalmente indígena y campesina (Dessaint, 1962, p. 347), capaz de ser movilizada a las plantaciones justo en los períodos intensos de cosecha. Paralelamente, en las haciendas conocidas como "fincas", se erguían las rancherías en donde vivía otro grupo de trabajadores permanentes, semiasalariados, mestizos y con funciones polivalentes, llamados “mozos colonos". Mientras esto sucedía, avanzaba "lentamente la mecanización" (Adams, 1956, p. 89), hasta que en los años 60 y 70 se introdujo nueva tecnología, pero las diferencias entre trabajadores de la costa sur, continuaban marcadas por el sustrato étnico (Oglesby, 2002, p. 4) y lingüístico.

En los años 80, Guatemala estaba inmersa en una crisis política (Gramajo, Ponciano, \& Vandeveire, 2016), en parte por las relaciones obrero-patronales y campesino-tenencia de la tierra, que restringían la plena incorporación del país en el mercado global competitivo. Sin embargo, a partir de los 90, el modelo agroex- portador llevó a cabo alianzas estratégicas, fusiones empresariales y aumento de las privatizaciones, iniciando con ello una mayor reestructuración del trabajo, hasta que en el 2000 aumentó la subcontratación, el outsourcing, la desaparición del "mozo colono" (Herrera, 2000), y la consolidación de un trabajador agrícola "voluntario", completamente asalariado y urbanizado.

Es así, que, a partir de las dos últimas décadas del siglo XXI, en el área rural de la costa sur de Guatemala, se observa una tendencia hacia la diferenciación de trabajadores agrícolas entre: (1) un núcleo periférico, completamente rural, en condiciones precarias de salud y alimentación, voluminoso, migrante, prescindible, cuya competitividad consiste en sus cualidades físicas; y (2) un núcleo central, semipermanente por el lugar de su residencia cada vez más urbanizada (Olayo, 1994), semicalificado. pero capacitado en las nuevas tecnologías de la producción, resistente físicamente, temporal, pero igualmente prescindible.

Entre ambos núcleos, las diferencias étnicas parecen ser cada vez más tenues, pero más profundas las que se basan en la tecnificación, el rendimiento y la competitividad, es decir en la sustitución de los "haceres por los saberes". Esto ha generado nuevas formas de trabajo incluido el trabajo autorregulado, la gestión del tiempo y hasta los recursos personales para responder de manera flexible a las nuevas actividades laborales, formas de empleo y de ingresos no laborales.

Ante las contradicciones de los mercados de trabajo, la responsabilidad social empresarial surgió como un catalizador del posible riesgo social (Beck, 1998; Friedman, 2005) que pudiera afectar la intensidad y modernidad con que se produce en la costa sur de Guatemala. Es por ello que, con el propósito de incrementar la productividad, pero con "una conducta empresarial apegada a la ley y a la sociedad", la responsabilidad social empresarial en Guatemala fue "impulsada entre 1997 y 1998 por representantes del Banco Mundial" (Sánchez-Latour, 2010, p. 193). Esta visión de cambio corresponde al neoliberalismo económico en donde el empresariado emerge como actor social responsable (Jonas, 2004; Kliksberg \& Tomassini, 2000), que con renovada visión promueve la descentralización de los servicios públicos de salud por medio de alianzas público-privadas y de modelos autofinanciables de jornadas médicas, salud preventiva, programas nutricionales, saneamiento básico, capacitación, y empleo propio, entre otros servicios en beneficio de trabajadores directos e indirectos. En ese contexto, surgen las nuevas alianzas de desarrollo y construcción de capital social con una 
lógica de excelencia empresarial en las regiones agroindustriales exportadoras.

En parte, esto tiene que ver con lo que explica Sánchez-Latour (2010, p. 451), de que luego del estallido social de la década de los ochenta (Forrester, 2011), los contingentes de trabajadores dejaron de ser solo un número, lo cual implicó modificar la forma de relacionarse con ellos, y así surgieron programas de orientación sobre enfermedades ocupacionales y sociales (entre ellas las de transmisión sexual), alimentación diseñada dietéticamente, cursos sobre primeros auxilios, manejo de herramientas de trabajo, insolación/ rehidratación, mordeduras de serpientes, higiene personal, entretenimiento, etc.

Según Herrera-Ruiz (2016) con los nuevos modelos gerenciales del trabajo y de la responsabilidad social empresarial, se trazó el camino a la competitividad sin dejar espacio a los antagonismos que generalmente se manifiestan en las relaciones laborales y patronales. Sin embargo, estos modelos aplican a los grandes consorcios agroindustriales, no así a las plantaciones proveedoras individuales que, para ser competitivas, funcionan al margen de la modernización productiva y de esas nuevas formas de administración de la fuerza laboral. No obstante, en ambos modelos de management del trabajo, se contratan grupos pequeños de trabajadores organizados en frentes de trabajo, que realizan variadas actividades agrícolas, durante períodos cortos de rotación en las diferentes plantaciones.

\section{EI trabajo en la determinación de la salud}

Entre los consorcios agroindustriales existe competencia por obtener mano de obra calificada, masculina, resistente físicamente, en edades comprendidas entre los 18 y 50 años. Así también son competitivos los incentivos laborales y el pago por productividad. Esto garantiza el incremento de la superficie cosechada y por supuesto, el incremento de los rendimientos que hacen del sector agroindustrial de la costa sur en uno de los modelos productivos más exitosos del agro centroamericano, y posiblemente latinoamericano, al predicar el incremento de la productividad a través de la tecnificación, la reconfiguración de los mecanismos de captación, administración, remuneración y control del trabajo.

Respecto a la remuneración, el salario promedio mensual en las actividades de campo para el algodón es de aproximadamente US\$130, en el corte de café es de US\$260 y para la caña de azúcar es de US\$208 a
US\$526. La zafra no implica un compromiso salarial mensual, ya que depende de la cantidad de toneladas cortadas diariamente que por lo menos debe alcanzar entre 3 y 4 TM. Sin embargo, para superar el salario mínimo establecido para el campo, el corte de caña necesita alcanzar $6 \mathrm{TM}$ en jornadas de entre 10 y $12 \mathrm{~h}$ diarias, con más o menos $20 \mathrm{~min}$ de descanso o para ingerir alimentos.

Aunque temporal, el pago monetario es un gran alivio para las economías familiares, no obstante, el considerable desgaste físico en los trabajadores agrícolas. Algunos de ellos a partir de los 40 años, explican que sus cuerpos empiezan a resentir la actividad física intensa y las enfermedades laborales asociadas. Así, por ejemplo, aunque la quema de caña ahora es controlada con métodos modernos, entre las poblaciones cercanas a los cultivos y específicamente entre los trabajadores se siguen reportando alergias, infecciones respiratorias, bronquitis y conjuntivitis como las enfermedades más frecuentes. Aunque no es determinante, en lugares como el municipio de Masagua también se han reportado casos aislados de leptospirosis.

En ese contexto, en los últimos 20 años, se empezaron a reportar casos de enfermedad renal crónica (ERC) que no estaban asociados con los factores tradicionales de riesgo, tal como diabetes mellitus (DM) e hipertensión arterial (HA) (Ferreiro et al., 2016). Estos reportes, tampoco estaban asociados con la edad, ya que empezaron a surgir en hombres muy jóvenes y niños que forman parte de lo que las normas internacionales establecen como "población que está apta en cuanto a edad para ejercer funciones productivas" (Instituto Nacional de Estadística [INE], 2019, p. 38). Para efectos de comparabilidad nacional, la edad de la población en edad de trabajar (PET) "se toma a partir de los 10 años o más y para el ámbito internacional a partir de los 15 años o más" (INE, 2019, p. 38).

Al respecto de la ERC, estudios como los de Harrison (2017) en toda la costa sur de Centroamérica, llamaron la atención sobre el estrés térmico asociado a la deshidratación en la actividad física intensa y que por la celeridad en que se manifiestan los síntomas puedan incidir en la enfermedad renal crónica no tradicional (ERCnT) que algunos investigadores también han dado por llamar Nefropatía Mesoamericana.

Mesoamérica (Kirchhoff, 1943) es un concepto histórico y cultural, pero también económico y ambiental que comprende desde la frontera septentrional de México hasta la frontera oriental de Panamá. De manera general, toda la parte sur de Mesoamérica, incluida 
Guatemala, concentra las tierras más ricas y aptas para la producción agrícola especialmente en la costa sur que en Guatemala que registra temperaturas promedio de más de $35^{\circ} \mathrm{C}$ (Instituto Privado de Investigación sobre Cambio Climático, 2019) que, en jornadas de trabajo continuas, incide en que la sensación térmica sea considerablemente mayor en las tareas de campo sin sombra.

En las plantaciones de los grandes consorcios agroindustriales, desde hace más de dos décadas existen programas de rehidratación por medio de sueros orales. Pero por lo que se ha podido monitorear, estos sueros llevan a la saciedad y a la falta de gusto. Para alguien sediento es más apetecible una bebida carbonatada o un refresco frio, pero sin comprender que a la larga solo incrementa la deshidratación por sus altos contenidos de azúcar.

Aspectos como el anterior, pueden ser abordados con renovados enfoques sociológicos, políticos y económicos para adentrarse en los factores que aún no han sido considerados en la etiología de la ERCnT. Desde la antropología, uno de los aportes más valiosos es el involucramiento de la perspectiva de los actores sociales con nuevos enfoques epistemológicos que tomen en cuenta las subjetividades de los trabajadores, las cuales se presentan como vida cotidiana (Lakoff \& Johnson, 2017) y forman parte de su "mundo coherente" (Berger \& Luckmann, 2003).

En el mundo de los trabajadores, la preocupación de los individuos es cómo insertarse y ajustarse a los nuevos requerimientos del mercado de trabajo, es por ello que los trabajadores rurales elaboran una serie de aspectos "subjetivos" que le dan coherencia a su mundo laboral y se manifiestan "objetivos" en su vida social. Al respecto autores como Bauman (2005) explican que esos nuevos esquemas de trabajo traen consigo exigencias más elaboradas y requieren otras formas de organizarse.

En esas formas de organizarse, el cambio social es un fenómeno colectivo que afecta a un importante conjunto de individuos. Al respecto Durkheim (1995) reconoció la integración y la crisis social a partir de la división del trabajo en la cohesión y el cambio social. A partir de su concepto de 'anomia' o debilitamiento de la moralidad común, explicó los cambios producidos en la división del trabajo en la sociedad y en sus modos de vida. En ese sentido la modernización supone cambio social, entendiendo éste como una modificación o variación de las estructuras sociales que se hallan incorporadas a normas, valores, productos y símbolos culturales, las que determinan las estrategias y respuestas de los trabajadores en el mundo laboral.

\section{El trabajo en la determinación social de las es- trategias y respuestas laborales}

En la confrontación de la división social del trabajo surgen nuevas doctrinas del trabajo: (1) las que subordinan el trabajo al mercado, negando las dimensiones políticas (Beck, 2004) de participación social y del Estado; (2) las que buscan desregular el trabajo a través de un nuevo acuerdo entre el capital y el trabajo con flexibilidad, y (3) las que hacen desaparecer las actividades hasta hace poco tradicionales en donde el "no trabajo" se aplica solo para una pequeña cantidad de personas, mientras que la mayoría trabaja con más intensidad por cortos períodos de tiempo, o bien oscilan entre el subempleo y desempleo. Esto implica que el trabajo se enfrenta a posturas disímiles con respecto a su transformación y disolución.

En la "crítica a un mundo sin trabajo", tal como ha propuesto Beck (2004, p. 47), la peor angustia es la falta de empleo (Forrester, 1997) y por supuesto remuneración para cubrir por lo menos la canasta básica alimentaria (CBA) que en Guatemala a diciembre del 2019 era de Q.3,58491.00 (US\$465.57) (INE, 2020), contrastado con un salario mínimo para actividades agrícolas de Q.2,992.37 (US\$388.62), y para actividades exportadoras y de maquila de Q.2,831.77 (US\$367.76) (Ministerio de Trabajo, 2020).

El estrés que genera la falta de empleo, por conservar la fuente de trabajo, o por no ser despedido, puede desencadenar trastornos físicos, psicológicos y por qué no decirlo también sociales, como factores determinantes de la salud en extensiones territoriales tan bastas como especializaciones productivas existen. En este sentido la ERCnT "es un factor enorme de morbilidad y mortalidad en los países sin otras oportunidades de empleo, y por eso es tan devastador en las comunidades que están siendo afectadas" (Harrison, 2017, p. 3).

\section{Agradecimientos}

A Pedro Costa Morata por su dirección y constante ayuda en la preparación de en mi tesis doctoral presentada en la Facultad de Ciencias Políticas y Sociología de la Universidad Pontificia de Salamanca. Así también a. Armando Cáceres por su minuciosa dedicación en la revisión de un fragmento de esa tesis y por la motivación puesta en la publicación de los resultados. A Oliverio 
Juárez y Romeo Juárez que demasiado pronto dejaron de trabajar en la costa.

\section{Referencias}

Adams, R. (1956). Encuesta sobre la cultura de los ladinos en Guatemala. Título original de la obra "Culture Survey of Central América" (inédita). Versión castellana de Joaquín Noval. Editor: Jorge Luis Arriola. Guatemala: Editorial del Ministerio de Educación Pública.

Bauman, Z. (2005). Modernidad líquida. Buenos Aires: Fondo de Cultura Económica.

Beck, U. (1998). La sociedad del riesgo. Hacia una nueva modernidad. Barcelona: Paidós.

Beck, U. (2004). Capitalismo sin trabajo, sobre mitos políticos, la economía global y el futuro de la democracia. En L. Álvarez (Coord.), Un mundo sin trabajo. México: Driada.

Berger, P. \& Luckmann, T. (2003). La construcción social de la realidad. Buenos Aires: Amorrortu

Dessaint, A. (1962). Papel que juegan la hacienda y la plantación en el cambio socio-cultural: Guatemala y Brasil. Guatemala Indigena, 2 (abril-junio), 1769.

Durkheim, É. (1995). La división del trabajo social. Madrid: Akal.

Fausto, M. (2017). El mercado laboral rural en Guatemala: una perspectiva desde los derechos humanos. En K. Winkler, La vida campesina en el siglo XXI. Revista Territorios No 12. Congecooop

Ferreiro, A., Álvarez-Estévez, G., Cerdas-Caderón, M., Cruz-Trujillo, Z., Mena, E., Reyes, M., ... Ordúnez, P. (2016). Confirmed clinical case of chronic kidney disease of nontraditional causes in agricultural communities in Central America: a case definition for surveillance. Revista Panamericana de Salud Pública, 40(5), 301-308.

Friedman, M. (2005). The social responsibility of business is to increase its profits. En S. CollinsChobanian (Ed.), Ethical Challenges to Business as Usual (pp. 224-229). Upper Saddle River, New Jersey: Pearson, Prentice-Hall.

Foucault, M. (1968). Las palabras y las cosas. Madrid: Siglo XXI.
Forster, C. (2011). Miles de machetes en alto: las luchas campesinas de la costa sur en el surgimiento de la revolución guatemalteca, 1970-1980. En M. Vela. Guatemala, la infinita historia de las resistencias. Guatemala: Magna Terra editores.

Forrester, V. (1997). El horror económico. (Trad. D. Zadunaisky). Buenos Aires: Fondo de Cultura Económica.

Geertz, C. (1988). El antropólogo como autor. Barcelona: Paidós Studio.

Gramajo, L; Ponciano, K; \& Vandeveire, J. (2016). Lucha campesina y trabajo pastoral en la costa sur de Guatemala. Guatemala: Editorial Serviprensa.

Hocsman, L. (2015). Agricultura familiar y descampesinización. Nuevos sujetos para el desarrollo rural modernizante. Perspectivas Rurales. Nueva época, 13(25), 11-27.

Harrison, P. (2017). Brote de muertes por insuficiencia renal atribuidas a estrés térmico por actividad fisica intensa. Recuperado de Medscape. https:// espanol.medscape.com/verarticulo/5901779

Herrera, S. (2000). Azúcar y transformación del colonato como práctica económica-social y referente identitario en mozos de rancherías de la costa sur de Guatemala (Tesis de licenciatura). Universidad de San Carlos de Guatemala, Guatemala.

Herrera-Ruiz, S. (2016). De la finca a la empresa. Azúcar, poder, cambios económicos y transformación cultural en la costa sur de Guatemala (Tesis de doctorado). Universidad Pontificia de Salamanca. Madrid.

INE. (2019). Encuesta Nacional de Empleo e Ingresos. Principales Resultados. Recuperado de https:// www.ine.gob.gt/sistema/

Instituto Privado de Investigación sobre Cambio Climático. (2019). Resumen meteorológico 2018 del sur de Guatemala. Programa de Investigación en clima e Hidrología. Guatemala.

Jonas, H. (2004). El principio de responsabilidad. Ensayo de una ética para la civilización tecnológica. Barcelona: Herder.

Kirchhoff, P. (1943). Mesoamérica, sus límites geográficos, composición étnica y caracteres culturales. Acta Americana, 1, 92-107. 
Kliksberg, B., \& Tomassini, L. (2000). Capital social y cultura: Claves estratégicas para el desarrollo. Buenos Aires: Banco Interamericano de Desarrollo, Fondo de Cultura Económica.

Lakoff, G. \& Johnson, M. (2017). Metáforas de la vida cotidiana. Madrid,: Editorial Cátedra.

Ministerio de Trabajo. (2020). Salario mínimo de Guatemala. Recuperado de https://www. mintrabajo.gob.gt/index.php/dgt/salario-minimo

Oglesby, E. (2002). Elites, labor and Agrarian Modernization in Guatemala, 1980-2000 (PhD Thesis in Geography). University of California, Berkeley.

Olayo, L. (1994). Esquema preliminar de crecimiento urbano para la Ciudad de Santa Lucía Cotzumalguapa (Tesis de licenciatura). Universidad de San Carlos de Guatemala, Facultad de Arquitectura.

Prakash Sethi, S. (2003). Globalization and the good corporation: A need for proactive coexistence. Journal of Business Ethics, 43(1/2), 21-31.
https://doi.org/10.1023/A:1022954930225

Roberts, B. (ed.) (1998). Ciudadanía y política social. Colección Centroamérica en reestructuración. No. 3. San José, Costa Rica: Flacso,

Samuelson, P. \& Nordhaus, W. (2002). Economía. XVII edición. Madrid: McGraw-Hill/Interamericana de España.

Sánchez-Latour, A. (2010). Alianzas estratégicas, responsabilidad social empresarial y su impacto en los sectores más pobres de Guatemala (Tesis de doctorado). Universidad Pontificia de Salamanca. Facultad de Ciencias Políticas y Sociología "León XIII". Madrid, España.

Taylor, S. \& Bogdan, R. (1996). Introducción a los métodos cualitativos de investigación. La búsqueda de significados. Barcelona: Paidós. 